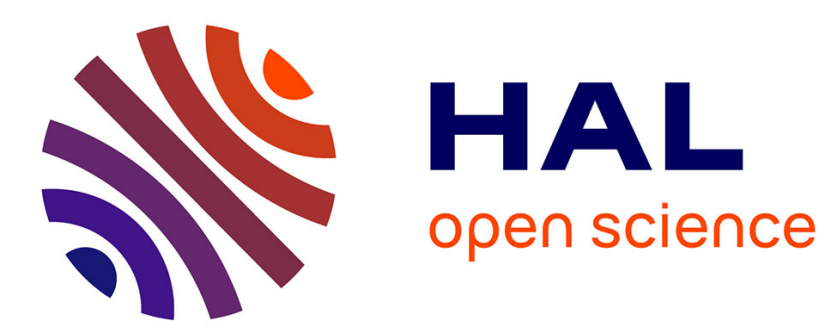

\title{
Boundary Geometric Control of a Nonlinear Diffusion System with Time-Dependent Spatial Domain
}

\author{
Ahmed Maidi, Jean-Pierre Corriou
}

\section{To cite this version:}

Ahmed Maidi, Jean-Pierre Corriou. Boundary Geometric Control of a Nonlinear Diffusion System with Time-Dependent Spatial Domain. Asian Journal of Control, 2015, 00 (4), pp.1 - 10. 10.1002/asjc.0000 . hal-02383518

\section{HAL Id: hal-02383518 https://hal.science/hal-02383518}

Submitted on 2 Dec 2019

HAL is a multi-disciplinary open access archive for the deposit and dissemination of scientific research documents, whether they are published or not. The documents may come from teaching and research institutions in France or abroad, or from public or private research centers.
L'archive ouverte pluridisciplinaire HAL, est destinée au dépôt et à la diffusion de documents scientifiques de niveau recherche, publiés ou non, émanant des établissements d'enseignement et de recherche français ou étrangers, des laboratoires publics ou privés. 


\begin{abstract}
A Stefan problem represents a distributed parameter system with a time-dependent spatial domain. This paper addresses the boundary control of the position of the moving liquid-solid interface in the case of nonlinear Stefan problem with Neumann actuation. The main idea consists in deriving an equivalent linear model by means of Cole-Hopf tangent transformation, i.e. under a certain physical assumption, the original nonlinear Stefan problem is converted to a linear one. Then, the geometric control law is deduced directly from that developed, by the authors of the present paper, for the linear Stefan problem. Based on the fact that the Cole-Hopf transformation is bijective, it is shown that the developed control law yields a stable closed-loop system. The performance of the controller is evaluated through numerical simulation in the case of stainless steel melting characterized by a temperature-dependent thermal conductivity, which is nonlinear. The objective is to control the position of the liquid-solid interface by manipulating a heat flux at the boundary.
\end{abstract}




\title{
Boundary Geometric Control of a Nonlinear Diffusion System with Time-Dependent Spatial Domain
}

\author{
Ahmed MAIDI $^{a}$, and Jean-Pierre CORRIOU ${ }^{b *}$ \\ ${ }^{a}$ Laboratoire de Conception et Conduite des Systèmes de Production, \\ Université Mouloud MAMMERI, 15000 Tizi-Ouzou, Algérie. \\ ${ }^{b}$ Laboratoire Réactions et Génie des Procédés, \\ UMR 7274-CNRS, Lorraine Université, ENSIC \\ 1, rue Grandville, BP 20451, 54001 Nancy Cedex, France.
}

December 2, 2019

Keywords: distributed parameter system, Cole-Hopf tangent transformation, Stefan problem, boundary control.

\section{Introduction}

Physical systems involving phase change phenomenon are characterized by a moving interface that separates the solid and liquid phases [1]. To cite a few, melting, solidification, casting, chemical reaction, heat and mass transfer belong to this class of systems, which is commonly termed Stefan problems in the literature $[1,2]$. The characteristic variables of this kind of systems depend both on space and time variables. These systems are classified as distributed parameter systems (DPSs), which are of infinite dimension and characterized by a time-dependent spatial domain [3, 4].

The dynamic behavior modeling of Stefan problems leads to a hybrid model $[1,5]$ involving coupled partial and ordinary differential equations

\footnotetext{
*Corresponding author: jean-pierre.corriou@univ-lorraine.fr
} 
together with initial and boundary conditions. The partial differential equations (PDEs) describe the system dynamic behavior while the movement of the solid-liquid interface, that forms actually a moving boundary of the system, is modeled by an ordinary differential equation (ODE).

The control of DPSs represents a very challenging field and occupies an important place in control theory [6]. Note that most contributions are based on the early lumping approach $[7,8]$, i.e. the controller design is achieved using an equivalent ODEs model, denoted as a reduced model, that approximates the original PDEs one. This reduced model is derived using some powerful reduction techniques that achieve an accurate approximation characterizing the dominant dynamic behavior of the distributed parameter system (DPS) described by the PDEs model [9]. This reduced model is obtained by approximating either the PDEs (for instance by discretization using either finite differences or finite elements methods) or their solutions (using for example Galerkin's method). This design procedure, denoted as early lumping approach, leads to a high dimension controller with a limited performance due to the approximation process that masks the spatially distributed nature of the DPS [6, 10]. Nevertheless, during these last years, significant efforts have been made to develop sophisticated control strategies that enhance significantly the closed-loop performance following the early lumping approach $[11,12]$.

An interesting alternative to the early lumping approach is the late lumping approach that consists in designing the controller using directly the PDEs model without any approximation [7] The late lumping approach preserves the spatially distributed nature and the fundamental control theoretical properties of the DPS, which allows to increase the controller performance $[6,8]$. Several successful applications of the late lumping are reported in the literature $[6,13,14,15,4]$. Nevertheless, most developed control strategies and applications assume a fixed spatial domain, hence control of DPSs with a time-dependent domain constitutes a very challenging field. In this area, few contributions are reported in the literature $[16,17,3,18,19,20]$ and most of them are based on the early lumping approach [16, 17, 18].

The Stefan problem has been investigated in literature and, in most contributions, it is formulated as an inverse geometry problem [3, 21, 22] that is solved in open loop. The approach developed here is based on closed-loop control and thus differs by a large extent. To the best of our knowledge, the first application of the late lumping approach to the DPSs with timedependent spatial domain has been done by the authors of the present paper 
in the case of a linear Stefan problem [4]. In their contribution, the authors tackle the closed-loop control of a linear Stefan problem, that is, a linear diffusion process with a time-dependent spatial domain. Thus, a boundary geometric control law that enforces the solid-liquid interface position tracking was developed using the concepts of characteristic index $[6,8]$ and the closed-loop stability was demonstrated using the Lyapunov stability test. The performance of the developed control law was evaluated by simulation in the case of a zinc melting process.

In recent years, geometric control emerged as an interesting and suitable approach for designing controllers for DPSs using the late lumping approach [6, 23, 24, 13, 4]. Geometric control presents the following advantages:

- the controller can be easily designed using the PDE model by performing only successive time derivatives of the controlled variable $[6,13,4]$,

- it allows the use of the full potential of an existing control theory for lumped linear systems that offers powerful controller design techniques to enhance the performance of the obtained linearized system [6, 23, 24],

- in the case of linear DPSs, it is easy to show, using some concepts from semigroup and Lyapunov theories, the stability of closed-loop system [6, 13, 4].

The advantages cited above and the results developed by the authors of the present paper in the case of linear Stefan problem [4] motivate the extension of the geometric control to a nonlinear diffusion process with timedependent domain. Thus, based on their original results, the authors address the control of the solid-liquid interface position in the case of a nonlinear Stefan problem. It is shown that by using the Cole-Hopf tangent transformation [25, 26], an equivalent PDE linear model of the nonlinear Stefan problem can be easily derived. The resulting control law, that enforces position tracking of the liquid-solid interface, of the assumed nonlinear Stefan problem can be derived directly from the control law of the linear Stefan problem. Then, based on the fact that the Cole-Hopf tangent transformation is bijective, it is demonstrated that the obtained controller yields a stable closed-loop system. The performance of the developed controller is shown by numerical simulation in the case of melting of stainless steel characterized by a temperature-dependent thermal conductivity, which makes it a nonlinear diffusion process. The objective is to control the position of the liquid-solid interface by manipulating a heat flux applied at the boundary.

The paper is structured as follows: Section 2 is devoted both to the general Stefan problem and to the control problem formulation. The mathematical model of the Stefan problem is presented in Section 3. Section 4 gives 
the geometric control law in the case of a linear Stefan problem. The boundary geometric of the nonlinear Stefan problem is investigated in Section 5 . The performance of the controller is evaluated, by numerical simulation, in Section 6 in the case of stainless steel melting. Section 7 concludes the paper.

Note that the present study addresses the control of a nonlinear diffusion with a time-dependent space domain, thus without loss of generality, the terminology from heat conduction transfer will be used in this paper to simplify the presentation.

\section{Stefan problem and control problem for- mulation}

A schematic representation of a melting process of a finite strip of a solid material of length $L$ is given in Figure 1. The heat flux $\dot{q}(t)$ applied at the left boundary $z=0$ causes the melting process that moves in the positive $z$ direction. The system is thus composed of two phases, liquid and solid, separated by a moving liquid-solid interface. At the position $z=l(t)$ corresponding to the liquid-solid interface, the temperature of the liquid is equal to the melting temperature $T_{f}$ of the considered material.

In the Stefan problem, the aim is to move the liquid-solid interface $l(t)$ to a desired position $l^{d}(t)$ by manipulating the heat flux $\dot{q}(t)$ at the boundary $z=0$. Thus, the control problem consists in designing a control profile $\dot{q}(t)$ that ensures this objective in a finite time. The design of this control law needs to set the formulated control problem under a mathematical model form which is given in the following Section.

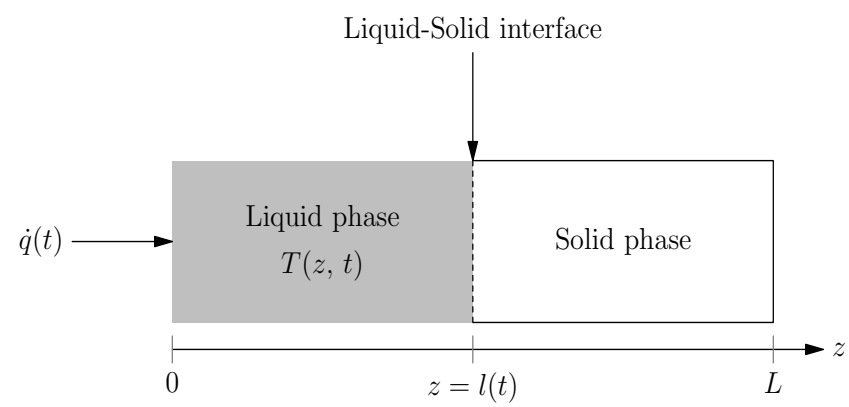

Figure 1: Schematics of material melting. 


\section{Mathematical model of Stefan problem}

Assuming that the heat conductivity of the liquid phase is temperaturedependent, the evolution of the liquid phase temperature is modeled by the following nonlinear diffusion equation [5]

$$
\rho c_{p} \frac{\partial T(z, t)}{\partial t}=\frac{\partial}{\partial z}\left(k(T(z, t)) \frac{\partial T(z, t)}{\partial z}\right)
$$

with the following fixed boundary conditions

$$
\begin{aligned}
-\left.k(T(0, t)) \frac{\partial T(z, t)}{\partial z}\right|_{z=0} & =\dot{q}(t) \\
T(l(t), t) & =T_{f}
\end{aligned}
$$

and the initial condition

$$
T(z, 0)=T_{0}(z)
$$

with $0 \leq z \leq l(t) \leq L$. The PDE (1) is quasilinear, that is a kind of nonlinear PDEs [27].

The motion of the liquid-solid interface is described by the following ODE $[1,5]$

$$
\rho \Delta H_{f} \frac{d l(t)}{d t}=-\left.k(T(l(t), t)) \frac{\partial T(z, t)}{\partial z}\right|_{z=l(t)}
$$

In this model, $T(z, t)$ is the temperature of liquid phase, $z$ the space variable, $t \in[0, \infty[$ the time variable, $l(t)$ the position of the moving boundary (liquid-solid interface). The spatial domain at time $t$ is denoted by $\Omega_{t}=$ $[0, l(t)] \subset \Omega$ with $\Omega=[0, L]$ the largest spatial domain, i.e., $0 \leq l(t) \leq L$. $\dot{q}(t)$ denotes the manipulated heat flux at boundary $z=0$. The parameters $\rho, c_{p}, k, \Delta H_{f}$ and $T_{f}$ are the liquid density, liquid specific heat, liquid heat conductivity, latent heat of fusion and melting temperature of the material, respectively.

Note that, in the case where both $c_{p}$ and $k$ are constant, i.e. independent of the temperature $T(z, t)$, the nonlinear PDE $(1)$ is reduced to a linear one. In this case, the control problem formulated in Section 2 has been solved by Maidi [4] in the framework of geometric control. The main results of their work used in the present study are summarized in the following section. 


\section{Geometric control of linear Stefan problem}

Assuming that $k$ is temperature-independent, i.e. constant, the nonlinear model (1)-(5) is reduced to the following linear model

$$
\begin{aligned}
\frac{\partial T(z, t)}{\partial t} & =\alpha \frac{\partial^{2} T(z, t)}{\partial z^{2}}, \quad \alpha=\frac{k}{\rho c_{p}} \\
-\left.k \frac{\partial T(z, t)}{\partial z}\right|_{z=0} & =\dot{q}(t) \\
T(l(t), t) & =T_{f} \\
T(z, 0) & =T_{0}(z) \\
\rho \Delta H_{f} \frac{d l(t)}{d t} & =-\left.k \frac{\partial T(z, t)}{\partial z}\right|_{z=l(t)}
\end{aligned}
$$

which corresponds to the linear Stefan problem. In this case, the control of the position of liquid-interface by manipulating the heat flux $\dot{q}(t)$ has been solved by the authors of the present paper [4]. To give more generality to their results, a dimensionless model of (6)-(10) has been used in both control design and closed-loop stability analysis. The dimensionless variables used are

$$
w(\xi, \tau)=\frac{T(z, t)-T_{\min }}{T_{\max }-T_{\min }}, \quad \tau=\frac{\alpha t}{L^{2}}, \quad \xi=\frac{z}{L}
$$

which yields the following dimensionless model

$$
\begin{aligned}
\frac{\partial w(\xi, \tau)}{\partial \tau} & =\frac{\partial^{2} w(\xi, \tau)}{\partial \xi^{2}}, 0 \leq \xi \leq l_{a}(\tau) \leq 1 \\
-\left.\frac{\partial w(\xi, \tau)}{\partial \xi}\right|_{\xi=0} & =\dot{q}_{a}(\tau), \quad \dot{q}_{a}(\tau)=\frac{L \dot{q}(t)}{k\left(T_{\max }-T_{\min }\right)} \\
w\left(l_{a}(\tau), \tau\right) & =w_{f} \\
w(\xi, 0) & =w_{0}(\xi) \\
\frac{\Delta H_{f}}{c_{p}} \frac{d l_{a}(\tau)}{d \tau} & =-\left.\left(T_{\max }-T_{\min }\right) \frac{\partial w(\xi, \tau)}{d \xi}\right|_{\xi=l_{a}(\tau)}
\end{aligned}
$$

where the dimensionless variables $w_{f}, w_{0}, \dot{q}_{a}$ and $l_{a}$ are the solid melting temperature, initial solid strip temperature, heat flux and liquid-solid interface position, respectively. $T_{\min }$ and $T_{\max }$ are arbitrary temperatures adequately chosen for dimensionless reasons. 
The control law developed by Maidi [4], in the framework of geometric control, using the concept of the characteristic index introduced by Christofides [6], is given as follows

$$
\begin{aligned}
\dot{q}_{a}(\tau)= & \frac{1}{\gamma}\left[w_{m}^{d}(\tau)-w_{m}(\tau)\right] \\
& -\left.\left[1-\frac{c_{p} w_{f}\left(T_{\max }-T_{\min }\right)}{\Delta H_{f}}\right] \frac{\partial w(\xi, \tau)}{\partial \xi}\right|_{\xi=l_{a}(\tau)}
\end{aligned}
$$

where $w_{m}(\tau)$ is an auxiliary output defined as the dimensionless spatial average temperature of the liquid phase, along the spatial domain $\Omega_{\tau}$, defined as follows

$$
w_{m}(\tau)=\int_{\Omega_{\tau}} w(\xi, \tau) d \xi
$$

which is introduced to ensure the controllability condition, i.e. to have a finite characteristic index $\sigma$ of the input-output system with input $\dot{q}(\tau)$ and the output $w_{m}(\tau)$ since, in the case of the output $l_{a}(\tau)$, the characteristic index $\sigma$ does not exist $(\sigma \rightarrow \infty)$. $\gamma$ and $w_{m}^{d}(\tau)$ are the dimensionless closedloop time constant and desired set point of the controlled output $w_{m}(\tau)$, respectively.

The developed control (17) yields the following closed-loop system

$$
\gamma \frac{d w_{m}(\tau)}{d \tau}+w_{m}(\tau)=w_{m}^{d}(\tau)
$$

and it is shown that, using the Lyapunov stability test, the internal dynamics is stable. Note that the developed control law (17) ensures the output tracking of the auxiliary variable $w_{m}(\tau)$ but our objective is to control the position of the liquid-solid interface $l_{a}(\tau)$. Hence, to solve the original control problem, a simple control strategy has been proposed based on the steady state relation between the spatial average temperature of the liquid phase $w_{m}(\tau)$ and the position of the liquid-solid interface $l_{a}(\tau)$.

In this section, the authors summarize only the necessary results that will be used later in the present study. Geometric control of a linear Stefan problem has been studied extensively by Maidi [4], thus more details about the derivation of the control law (17), the stability analysis of the resulting closed-loop system and the general control strategy proposed can be found in this original work. 
By assuming the dimensionless variables (11), the dimensional control law $\dot{q}(t)$ derived from the dimensionless control law (17) is

$$
\begin{aligned}
\dot{q}(t)= & \frac{\rho c_{p}}{\mu}\left[T_{m}^{d}(t)-T_{m}(t)-\right. \\
& \left.\left.\mu \alpha\left[1-\frac{c_{p} T_{f}}{\Delta H_{f}}\right] \frac{\partial T(z, t)}{\partial z}\right|_{z=l(t)}\right]
\end{aligned}
$$

where $\mu$ is the dimensional closed-loop time constant and $T_{m}(t)$ is the dimensional spatial average temperature of the liquid phase $T(z, t)$ defined as

$$
T_{m}(t)=\mathcal{C} T(z, t)=\int_{\Omega_{t}} T(z, t) d z
$$

and $T_{m}^{d}(t)$ is its desired set point. $\mathcal{C}$ is a bounded linear operator.

The results presented in this section are exploited to derive the geometric control law of the nonlinear Stefan problem (1)-(5), which is discussed in the following section.

\section{Geometric control of nonlinear Stefan prob- lem}

If the control theory of the linear DPSs has reached a certain level of maturity thanks to the well-established and powerful semi-group theory [28], the control theory of nonlinear DPSs is a challenging field with many interesting open questions [10]. Concerning the control problem of nonlinear DPSs, most contributions come from the early lumping approach [29, 30]. Recently, the late lumping approach has been applied, in the framework of geometric control, to control nonlinear DPSs and particularly nonlinear diffusion systems [13].

Tangent transformations play a key role in studying and solving nonlinear PDEs. They allow to convert nonlinear PDEs to linear ones [25]. For instance, under a certain assumption, the nonlinear diffusion equation (1) can be easily converted to a linear diffusion equation using the Cole-Hopf transformation [26]. This transformation has been exploited in the case of a nonlinear diffusion process, with a distributed control, to design a controller 
following the late lumping approach [13] and to study the controllability propriety of a nonlinear diffusion system [31]. In the following, the Cole-Hopf transformation is used to deduce the control of the nonlinear Stefan problem (1)-(5) from the control law (20) obtained in the case of the linear Stefan problem.

\subsection{Cole-Hopf Transformation}

The Cole-Hopf transformation consists in converting the nonlinear diffusion equation (1) into a linear one if the thermal diffusivity defined by the following ratio

$$
\alpha=\frac{k(T(z, t))}{\rho c_{p}}
$$

is approximately constant. This hypothesis is accepted in many cases since the variation of $\alpha$ with $T(z, t)$ is much less important than that of $k(T(z, t))$, so that this approximation is reasonable [32].

To linearize the nonlinear diffusion equation (1), using the Cole-Hopf technique, one seeks a transformation of the form [26]

$$
T(z, t)=\varphi(\theta(z, t))
$$

where $\varphi($.$) is a nonlinear function.$

From a physical point of view, the liquid phase temperature $T(z, t)$ cannot undergo discontinuities in a single-phase medium and, to each temperature $T(z, t)$, corresponds one temperature $\theta(z, t)$. Consequently, the nonlinear function $\varphi($.$) is a bijective mapping and both temperatures T(z, t)$ and

$\theta(z, t)$ are continuous functions with respect to $z$, that is, they are spatially differentiable.

By using the transformation (23), the evaluation of the derivatives of the left-hand side of (1) gives

$$
\frac{\partial T(z, t)}{\partial t}=\frac{d \varphi(\theta(z, t))}{d \theta(z, t)} \frac{\partial \theta(z, t)}{\partial t}
$$

and for the right-hand side of (1), one obtains

$$
\begin{aligned}
& \frac{\partial}{\partial z}\left(k(T(z, t)) \frac{\partial T(z, t)}{\partial z}\right)=M\left(\frac{\partial \theta(z, t)}{\partial z}\right)^{2} \\
& +k(\varphi(\theta(z, t))) \frac{d \varphi(\theta(z, t))}{d \theta(z, t)} \frac{\partial^{2} \theta(z, t)}{\partial z^{2}}
\end{aligned}
$$


with

$$
\begin{aligned}
M= & k(\varphi(\theta(z, t))) \frac{d^{2} \varphi(\theta(z, t))}{d \theta^{2}(z, t)} \\
& +\frac{d k(\varphi(\theta(z, t)))}{d \varphi(\theta(z, t))}\left(\frac{d \varphi(\theta(z, t))}{d \theta(z, t)}\right)^{2}
\end{aligned}
$$

To make the right-hand side of (25) linear, the term $M$ is set equal to zero. This term is a differential equation, which can be expressed in the following integrable form

$$
\frac{d}{d \theta(z, t)}\left[k(\varphi(\theta(z, t))) \frac{d \varphi(\theta(z, t))}{d \theta(z, t)}\right]=0
$$

Integrating (27) gives

$$
k(\varphi(\theta(z, t))) \frac{d \varphi(\theta(z, t))}{d \theta(z, t)}=c_{1}
$$

hence

$$
\begin{aligned}
\int k(\varphi(\theta(z, t))) d \varphi(\theta(z, t)) & =\int c_{1} d \theta(z, t) \\
& =c_{1} \theta(z, t)+c_{2}
\end{aligned}
$$

or equivalently

$$
\begin{aligned}
\theta(z, t) & =\varphi^{-1}(T(z, t)) \\
& =\frac{1}{c_{1}} \int k(T(z, t)) d T(z, t)-\frac{c_{2}}{c_{1}}
\end{aligned}
$$

where $\varphi^{-1}($.$) is the inverse function of \varphi($.$) and c_{1} \neq 0$. According to the discussion in Subsection 5.1, as the mapping $\varphi($.$) is a continuous bijective$ function, it follows that its inverse $\varphi^{-1}($.$) exists and is also a continuous$ function. The two arbitrary constants $c_{1}$ and $c_{2}$ can be chosen as follows [26]

$$
c_{1}=k(0) \quad ; \quad c_{2}=-\left[\int k(T) d T\right]_{T=0}
$$

Thus $c_{2}$ is the primitive integral of the heat conductivity $k(T)$ taken at $T=0$. This leads, as demonstrated by Vadasz [26], after some elementary mathematical manipulations, to the following well-known Kirchhoff's transformation

$$
\theta(z, t)=\varphi^{-1}(T(z, t))=\frac{1}{k(0)} \int_{0}^{T(z, t)} k(\zeta) d \zeta
$$


which is actually a particular case of Cole-Hopf transformation.

In summary, using the transformation (23), with $\varphi(\theta(z, t))$ satisfying (27) and considering the expression (22) of diffusivity $\alpha$, the nonlinear state equation (1) will be converted to the following linear one

$$
\frac{\partial \theta(z, t)}{\partial t}=\alpha \frac{\partial^{2} \theta(z, t)}{\partial z^{2}}
$$

and by taking into account both equations (28) and (31), the following new boundary and initial conditions can be easily deduced

$$
\begin{aligned}
-\left.k(0) \frac{\partial \theta(z, t)}{\partial z}\right|_{z=0} & =\dot{q}(t) \\
\theta(l(t), t) & =\varphi^{-1}\left(T_{f}\right)=\theta_{f} \\
\theta(z, 0) & =\varphi^{-1}\left(T_{0}(z)\right)=\theta_{0}(z)
\end{aligned}
$$

and the equation of the interface motion (5) takes the following form

$$
\rho \Delta H_{f} \frac{d l(t)}{d t}=-\left.k(0) \frac{\partial \theta(z, t)}{\partial z}\right|_{z=l(t)}
$$

Equations (33)-(37) represent a linear Stefan problem obtained by linearizing the nonlinear Stefan model (1)-(5) by means of Cole-Hopf transformation. Therefore, the control law of the nonlinear Stefan problem (1)-(5) can be deduced based on its equivalent linear model (33)-(37). This is explained in the following subsection.

\subsection{Control law and closed-loop stability analysis}

Based on the assumption made with respect to the diffusivity $\alpha$, given by (22), being nearly constant compared to the variation of heat conductivity $k$, the equivalent linear model (33)-(37) provided by the Cole-Hopf transformation can be used to design the control law of the nonlinear Stefan problem (1)-(5). Hence, according to the development given in Section 4, the con-

trol law that enforces the output tracking for the linear equivalent model 
$(33)-(37)$ is

$$
\begin{aligned}
\dot{q}(t)= & \frac{\rho c_{p}}{\mu}\left[\theta_{m}^{d}(t)-\theta_{m}(t)\right. \\
& \left.-\left.\mu \alpha\left(1-\frac{c_{p} \theta_{f}}{\Delta H_{f}}\right) \frac{\partial \theta(z, t)}{\partial z}\right|_{z=l(t)}\right]
\end{aligned}
$$

where the measured output is

$$
\theta_{m}(t)=\int_{\Omega_{t}} \theta(z, t) d z
$$

and $\theta_{m}^{d}(t)$ is its corresponding set point.

Thereafter, considering relations (30), (35) and (39), the control law (38) can be expressed according to the temperature $T(z, t)$ as follows

$$
\begin{aligned}
\dot{q}(t)= & \frac{\rho c_{p}}{\mu}\left[T_{m}^{d}(t)-\int_{0}^{l(t)} \varphi^{-1}(T(z, t)) d z\right. \\
& \left.-\left.\mu \alpha\left(1-\frac{c_{p} T_{f}}{\Delta H_{f}}\right) \frac{\partial\left[\varphi^{-1}(T(z, t))\right]}{\partial z}\right|_{z=l(t)}\right]
\end{aligned}
$$

which leads to the following closed-loop system

$$
\mu \frac{d T_{m}(t)}{d t}+T_{m}(t)=T_{m}^{d}(t)
$$

which is externally or BIBO-stable. Nevertheless, without the stability of the unobservable part, termed internal dynamics, the closed-loop stability of (41) is not guaranteed. In geometric control, the behavior of the internal dynamics, i.e. of the state of the system, plays a central role in proving the stability.

In the case of linear Stefan problem [4], based on the fact that the latent heat overcomes the sensible heat by a large extent for most physical systems, the authors demonstrated the stability of the internal dynamics of the closed-loop system using Lyapunov stability test. They concluded that the closed-loop system is exponential stable and $\theta(z, t) \rightarrow \theta_{f}$ when $t \rightarrow \infty$. Consequently, the exponential stability of the closed-loop system (41), that is the stability of its internal dynamics, can be deduced from the stability 


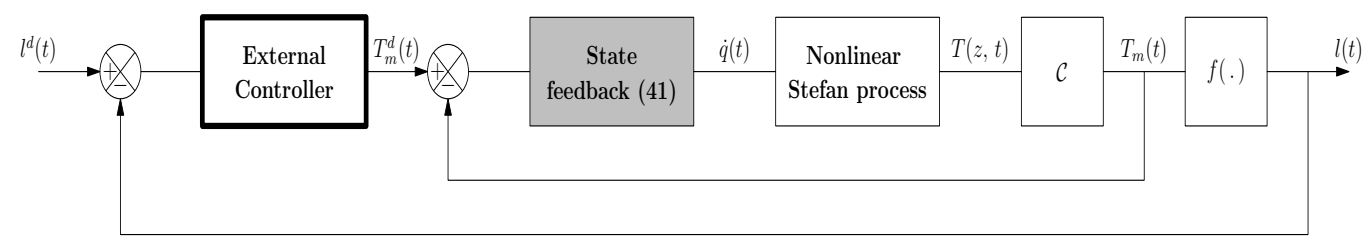

Figure 2: Boundary control strategy of the liquid-solid interface.

of its equivalent linear model (33)-(37) using the fact that Cole-Hopf transformation (23) is bijective. Lyapunov stability test for the equivalent linear model (33)-(37) yields that $\theta(z, t) \rightarrow \theta_{f}$ when $t \rightarrow \infty$, therefore using the Cole-Hopf transformation (23), it can be concluded that $T(z, T) \rightarrow \varphi\left(\theta_{f}\right)$ when $t \rightarrow \infty$, i.e. according to (35), it follows that $\lim _{t \rightarrow \infty} T(z, t)=T_{f}$. This means that the closed loop system (41) is internally stable.

\subsection{Global control strategy}

Recall that our objective consists in solving the initial boundary control problem, i.e., controlling the position of the liquid-solid interface $l(t)$ by manipulating the heat flux $\dot{q}(t)$ at the boundary $z=0$. Thus, it is proposed to keep the control law (40) derived for the auxiliary output (21) and to define its set point $T_{m}^{d}(t)$ by mean of an external controller taking as input the error $e(t)=l^{d}(t)-l(t)$, where $l^{d}(t)$ is the corresponding set point of the position $l(t)$ of the liquid-solid interface. The proposed global control strategy is shown in Fig 2.

The external controller in Fig. 2 is designed for the following nonlinear system

$$
\begin{aligned}
\dot{T}_{m}(t) & =-\frac{1}{\mu} T_{m}(t)+\frac{1}{\mu} T_{m}^{d}(t) \\
l(t) & =f\left(T_{m}(t)\right)
\end{aligned}
$$

Note that the bijective function $f($. ) can be identified by assuming several values of the desired set point $T_{m}^{d}(t)$ and their corresponding set points $l^{d}(t)$ (see the application example given is Section 6). 


\section{$6 \quad$ Melting of the stainless steel}

The tracking performance of the proposed control strategy is evaluated in the case of melting of a strip of stainless steel of length $l_{\max }=0.5 \mathrm{~m}$ the physical properties of which are given in Table 1 . The thermal conductivity $k$ is a nonlinear function of the temperature given as [33]

$$
k(T(z, t))=14.6+1.2710^{-2} T(z, t)
$$

where $T$ is expressed in ${ }^{\circ} \mathrm{C}$ and $k$ in $\mathrm{W} \cdot \mathrm{m}^{-1} \cdot \mathrm{K}^{-1}$. Thereafter, eq. (32) gives

$$
\varphi^{-1}(T(z, t))=T(z, t)+4.349310^{-4} T^{2}(z, t)
$$

Note that, for some complex thermal conductivity $k$, the analytical expression of $\varphi^{-1}($.$) cannot be calculated so that, in that case, numerical$ integration methods should be used.

For the closed-loop system simulation, the boundary immobilization method $[34,4]$ is used to derive an equivalent nonlinear model with an immobilized right-hand boundary condition for the original nonlinear system (1)-(5). Then, the equivalent nonlinear model obtained is simulated using the method of lines, based on finite differences, by taking 200 discretization points. The integral term of the control law (40) is evaluated using the trapezoidal method.

The relation, at steady-state, between the position of the liquid-solid interface position and the spatial average temperature $T_{m}(t)$ is given by Fig. 3 . From this figure, it follows that the relation between $l(t)$ and $T_{m}(t)$ is approximately linear, that is,

$$
T_{m}(t)=a l(t)
$$

\begin{tabular}{lll} 
Designation & Symbol & Value \\
\hline Density & & \\
Melting temperature & $T_{f}$ & $1648 \mathrm{Kg} \cdot \mathrm{m}^{-3}$ \\
Heat of fusion & $\Delta H_{f}$ & $260000 \mathrm{~J} \cdot \mathrm{kg}^{-1}$ \\
Heat capacity & $c_{p}$ & $490 \mathrm{~J} \cdot \mathrm{kg}^{-1} \cdot \mathrm{K}^{-1}$
\end{tabular}

Table 1: Stainless steel properties. 


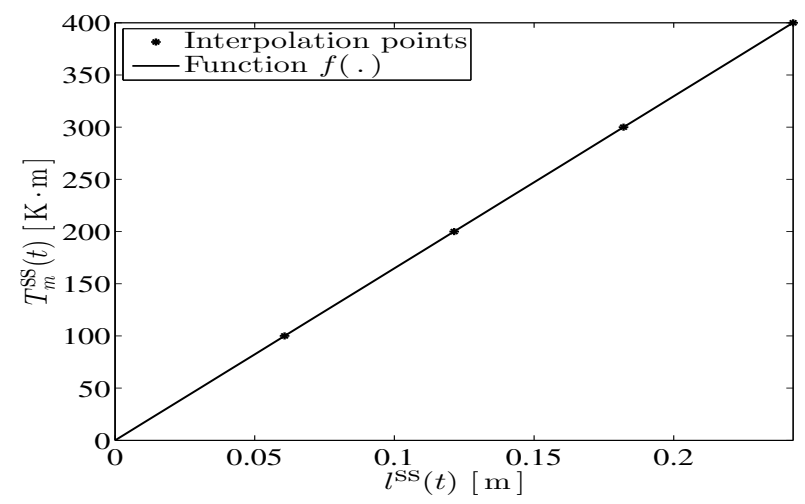

Figure 3: Steady-state relation between $T_{m}(t)$ and $l(t)$.

Hence, combining (41) and (46), the following transfer function between $T_{m}^{d}(t)$ and $l(t)$ results

$$
\frac{L(s)}{\tilde{T}_{m}^{d}(s)}=\frac{a}{\mu s+1}
$$

In this application, as the relation (46) is linear, hence the set point $T_{m}^{d}(t)$ is defined by means of a PI controller (external controller in Fig 2) as follows

$$
T_{m}^{d}(t)=K_{c}\left[\left(l^{d}(\zeta)-l(\zeta)\right)+\frac{1}{\tau_{i}} \int_{0}^{t}\left(l^{d}(\zeta)-l(\zeta)\right) d t\right]
$$

where $K_{c}, \tau_{i}$ and $l^{d}(t)$ are respectively the proportional gain, integral time constant and desired output of the liquid-solid interface position $l(t)$.

The controller tuning parameter $\mu$ is taken equal to $5 \mathrm{~min}$. The PI parameters are tuned so that the closed-loop system $l^{d}(t)-l(t)$ is characterized by a damping factor $\eta=1$ and a natural pulsation $\omega_{n}=0.18 \mathrm{rad} \cdot \mathrm{min}^{-1}$. The obtained parameters are $K_{c}=1318.8$ and $\tau_{i}=4.9383 \mathrm{~min}$. In order to have a smooth control signal, the desired set point $l^{d}(t)$ has been filtered by a second-order filter with time constants 1 min and 2 min.

In the following, the tracking capabilities of the proposed control strategy and its robustness against the parametric variation are investigated through numerical simulations. 


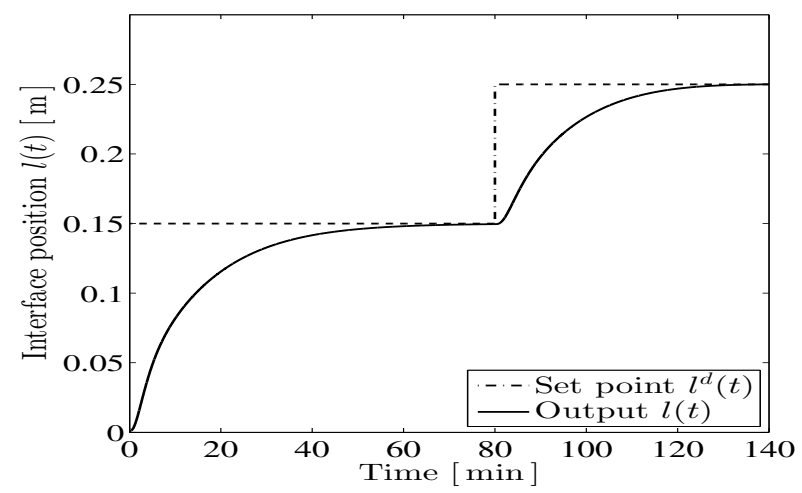

Figure 4: Tracking: Evolution of the position of the liquid-solid interface $l(t)$.

\subsection{Tracking problem}

The objective is to melt a portion of strip of length $0.3 \mathrm{~m}$, initially solid. To show the effectiveness of the developed controller, the melting process is achieved in two stages. First, a portion of $0.15 \mathrm{~m}$ is melted, once this is achieved, a new melting is started to melt the rest of the strip. Thus, two step set points $l^{d}(t)=0.15 \mathrm{~m}$ and $l^{d}(t)=0.30 \mathrm{~m}$ are imposed at $t=0$ and $t=$ 80 min, respectively. Fig. 4 shows that the imposed set points are perfectly tracked, i.e. the liquid-solid interface is moved to the specified positions. From Fig. 5, it follows that the heat flux evolution is physically acceptable and it is observed that the heat flux, initially zero, increases rapidly just after the variation of set point, then returns to its steady value as the liquid-solid interface reaches the specified positions.

\subsection{Robustness}

To evaluate the robustness of the controller, a perturbed model is simulated for a variation of the liquid specific heat $c_{p}$ assumed as parametric uncertainty. The robustness test performed consists in assuming that the control law (40) is calculated by assuming a constant liquid specific heat $c_{p}=454 \mathrm{~J} \cdot \mathrm{kg}^{-1} \cdot \mathrm{K}^{-1}$ whereas for the process is assumed to be temperaturedependent, that is, time-varying parameter. In this case, the thermal con- 


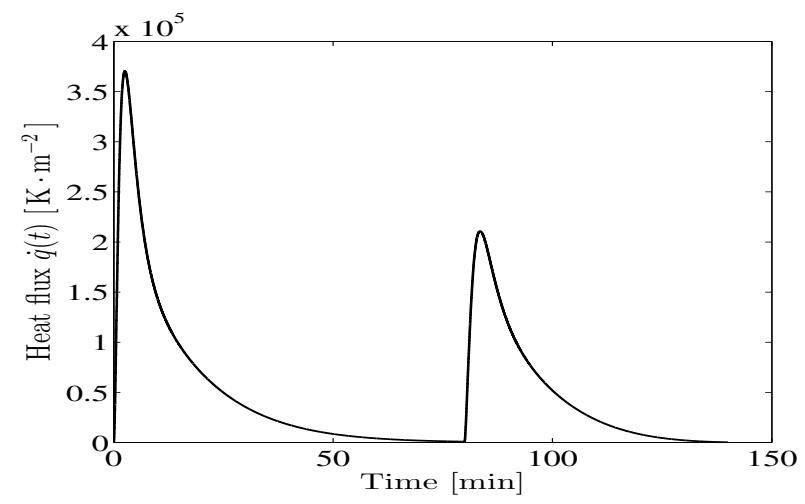

Figure 5: Tracking: Evolution of the manipulated heat flux $\dot{q}(t)$.

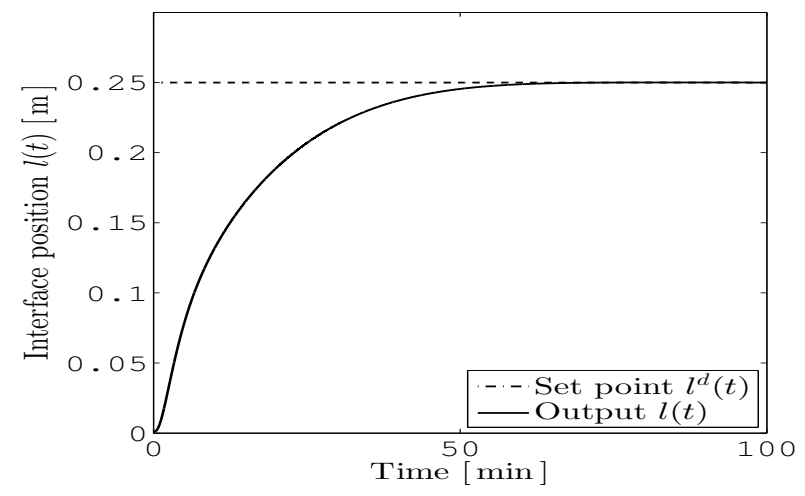

Figure 6: Robustness: Evolution of the position of the liquid-solid interface $l(t)$.

ductivity $c_{p}$ is a nonlinear function of the temperature $T(z, t)$ given as [33]

$$
\begin{aligned}
c(T(z, t))= & 454+0.388 T(z, t)+3.2210^{-4} T^{2}(z, t) \\
& +1.110^{-7} T^{3}(z, t) \quad\left(T \text { in }{ }^{\circ} \mathrm{C}\right)
\end{aligned}
$$

For this test, the objective is to melt a portion of strip, $0.25 \mathrm{~m}$ in length. Figures 6-7 show that in spite of the variation of $c_{p}$, the control objective is correctly achieved with admissible moves of the manipulated heat flux. 


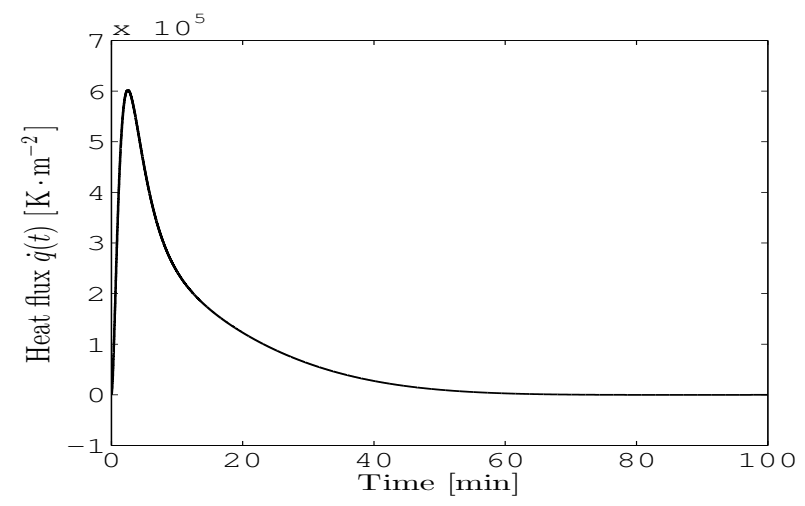

Figure 7: Robustness : Evolution of the manipulated heat flux $\dot{q}(t)$.

\section{Conclusion}

In this paper, a control strategy is proposed to control the position of the liquid-solid interface in a nonlinear Stefan problem. This is modeled by coupled partial and ordinary differential equations. The PDE describes the temperature evolution of the phase liquid, which is a nonlinear diffusion equation as the thermal conductivity and the specific heat are temperaturedependent. The motion of the liquid-solid interface is modeled by an ODE. The objective is to control the interface position by manipulating the heat flow at the opposite boundary.

Based on the tangent Cole-Hopf transformation, the control law of the nonlinear Stefan problem is deduced directly from the control law developed by the authors, in their previous work, for the control of Stefan linear problem. Thereafter, based on the fact that the Cole-Hopf transformation is a bijective mapping, it is shown that the developed control law yields a stable closed-loop system.

Finally, to achieve the initial objective control, i.e., to move the liquidsolid interface to a desired position, a control strategy is proposed where an external controller is introduced to define the set point of the spatial average temperature of the liquid phase. The error between the liquid-solid interface position and its desired set point is defined as the input of the external controller.

This control strategy is successfully tested by numerical simulation of the melting of a stainless steel strip. The obtained results show the ability of the controller to track the desired position of the interface with physically 
acceptable moves of the applied heat flux.

\section{References}

[1] Crank J. Free and moving boundary problems. Oxford University Press, 1984.

[2] Gupta S. The Classical Stefan Problem. Basic Concepts, Modelling and Analysis. Elsevier, 2003.

[3] Dunbar WB, Petit N, Rouchon P, Martin P. Motion planning for a nonlinear Stefan problem. ESAIM: Control, Optimisation and Calculus of Variations 2003; 9:275-296.

[4] Maidi A, Corriou JP. Boundary control of linear Stefan problem. J. Proc. Control 2014; 24(6):939-946.

[5] Mattheij RMM, Rienstra SW, ten Thije Boonkkamp JHM. Partial Differential Equations. Modeling, Analysis, Computation. SIAM, 2005.

[6] Christofides PD, Daoutidis P. Feedback control of hyperbolic PDE systems. AIChE J. 1996; 42(11):3063-3086.

[7] Ray WH. Advanced Process Control. Butterworths: Boston, 1989.

[8] Christofides PD. Nonlinear and robust control of PDE systems: methods and applications to transport-reaction processes. Birkhaüser: Boston, 2001.

[9] Li HX, Qi C. Modeling of distributed parameter systems for applications-A synthesized review from time-space separation. J. Proc. Control 2010; 20(8):891-901.

[10] Christofides PD. Control of nonlinear distributed process systems: Recent developments and challenges. AIChE J. 2001; 47(3):514-518.

[11] Pourkargar D, Armaou A. Modification to adaptive model reduction for regulation of distributed parameter systems with fast transients. AIChE J. 2013; 59(12):4595-4611. 
[12] Pourkargar D, Armaou A. Geometric output tracking of nonlinear distributed parameter systems via adaptive model reduction. Chem. Eng. Sci. 2014; 116(6):418-427.

[13] Maidi A, Corriou JP. Distributed control of nonlinear diffusion systems by input-output linearization. Int. J. Robust Nonl. Cont. 2014; 24(3):386-405.

[14] Shang H, Forbes JF, Guay M. Feedback control of hyperbolic distributed parameter systems. Chem. Eng. Sci. 2005; 60(4):969-980.

[15] Wu W, Liou C. Output regulation of nonisothermal plug-flow reactors with inlet perturbations. Comp. Chem. Engng 2001; 25(2-3):433-443.

[16] Armaou A, Christofides PD. Nonlinear feedback control of parabolic partial differential equation systems with time-dependent spatial domains. J. Math. Anal. and Appl. 1999; 239(1):124-157.

[17] Armaou A, Christofides PD. Robsut control of parabolic PDE systems with time-dependent spatial domains. Automatica 2001; 37(1):61-69.

[18] Ng J, Dubljevic S. Optimal boundary control of a diffusion-convectionreaction PDE model with time-dependent spatial domain: Czochralski crystal growth process. Chem. Eng. Sci. 2012; 67(1):111-119.

[19] Wang PKC. Stabilization and control of distributed parameter system with time-dependent spatial domains. J. Optimization Theory and Applications 1990; 65(2):331-362.

[20] Wang PKC. Feedback control of a heat diffusion system with timedependent spatial domains. Optimization Control: Applications and Methods 1995; 16(5):305-320.

[21] Khosravifard A, Hematiyan M, Wrobel L. Simultaneous control of solidus and liquidus lines in alloy solidification. Eng. Anal. with Boundary Elements 2013; 37(2):211-224.

[22] Nowak I, Smolkab J, Nowak A. Application of bezier surfaces to the 3-d inverse geometry problem in continuous casting. Inv. Prob. Sci. Eng. 2011; $\mathbf{3 7}(2): 211-224$. 
[23] Maidi A, Diaf M, Corriou JP. Boundary geometric control of a countercurrent heat exchanger. J. Proc. Control 2009; 19(2):297-313.

[24] Maidi A, Corriou JP. Distributed feedback design for systems governed by the wave equation. Int. J. Control 2011; 84(8):1417-1429.

[25] Meleshko SV. Methods for Constructing Exact Solutions of Partial Differential Equations. Mathematical and Analytical Techniques with Applications to Engineering. Springer: New York, 2005.

[26] Vadasz P. Analytical solution to nonlinear thermal diffusion: Kirchhoff versus Cole-Hopf transformations. J. Heat Transfer 2010; 132(12):121 302.1-121 302.6.

[27] Salsa S, Vegni F, Zaretti A, Zunino P. A Primer on PDEs. Models, Methods, Simulations. Springer: Italia, 2013.

[28] Pazy A. Semigroup of Linear Operators and Applications to Partial Differential Equations. Springer-Verlag: New York, 1983.

[29] Baker J, Christofides P. Finite-dimensional approximation and control of non-linear parabolic PDE systems. Int. J. Control 2000; 73(5):439-456.

[30] Dubljevic S, Christofides P, Kevrekidis I. Distributed nonlinear control of diffusion-reaction processes. Int. J. Robust Nonl. Control 2004; 14(2):133-156.

[31] Maidi A, Corriou JP. Controllability of a nonlinear diffusion system. Can. J. Chem. Eng. 2014; doi:DOI10.1002/cjce.22030.

[32] Carslaw HS, Jaeger JC. Conduction of Heat in Solids. Second edn., Oxford University Press: Oxford, UK, 1959.

[33] Taler J, Duda P. Solving Direct and Inverse Heat Conduction Problems. Springer: Berlin, 2006.

[34] Caldwell J, Ng DKS. Mathematical Modelling. Case Studies and Projects. Kluwer Academic Publishers, 2004. 